\title{
The Cauchy Integral Formula for Biregular Function in Octonionic Analysis
}

\author{
Yonghua Guo*, Haiyan Wang \\ School of Science, Tianjin University of Technology and Education, Tianjin, China \\ Email address: \\ 1974184907@qq.com (Yonghua Guo) \\ *Corresponding author
}

\section{To cite this article:}

Yonghua Guo, Haiyan Wang. The Cauchy Integral Formula for Biregular Function in Octonionic Analysis. International Journal of Theoretical and Applied Mathematics. Vol. 6, No. 3, 2020, pp. 39-45. doi: 10.11648/j.ijtam.20200603.12

Received: July 8, 2020; Accepted: August 21, 2020; Published: August 31, 2020

\begin{abstract}
In this paper, we mainly study the Cauchy integral formula and mean value theorem for biregular function in octonionic analysis. Octonion is the extension of complex number to non-commutative and non-associative space. Because of the non-associative properties of multiplication, octonion plays an important role in wave equation, Yang-Mills equations, operator theory and so on. In recent years, octonion has become a hot topic for scholars at home and abroad and got many rich results, such as Fourier transform, Bergman kernel, Taylor series and its applications in quantum mechanics. On the basis of two Stokes theorems, we get Cauchy integral formula for biregular function in octonionic analysis by using the methods in dealing with the Cauchy integral formula for biregular function in Clifford analysis and regular function in octonionic analysis. As a direct result we also get the mean value theorem for biregular function in octonionic analysis. This will generalize the corresponding conclusion in complex analysis and Clifford analysis, and lays a solid foundation for the application of octonionic analysis in physics.
\end{abstract}

Keywords: Octonion, Biregular Function, Cauchy Integral Formula, Mean Value Theorem

\section{Introduction}

Octonion is a generalization of quaternion to nonassociative algebra which has played a very important role in physical phenomena of black hole [1], supersymmetry and duality, extended supersymmetry [2], supergravity models etc [3-7]. Wang wei professor and his collaborators discussed the octonion Heisenber group [8]. Calin, O. and Chang, D. C. and Markina, I studied the geometric analysis on H-type groups related to division algebras [9]. In 1998, X. M. Li studied octonionic analysis [10]. Octonion has been widely studied by Baez [11]. Recently, Many experts and scholars are dedicated to octonionic analysis and obtain some results such as Cauchy integral formula for regular function, Hardy space, Bergman space [12-15]. H. Y. Wang and his collaborators studied the right inverse of Dirac in octonion space and generalized octonionic analysis to octonionic analysis of several variables [16-17]. J. X. Wang, X. M. Li described the octonion Bergman kernel for the unit ball [18]. In this paper, we will study regular function of two variables, called biregular function. But the octonions are neither commutative nor associative, which bring barriers to the study of the problems in biregular function. Therefore, we have biregular Cauchy integral formula and mean value theorem of octonions by use the associator to overcome the difficulties.

\section{Octonion}

The octonions $O$ are the nonassociative, noncommutative, normed division algebra over the real generated by $\mathrm{e}_{1}, \ldots, \mathrm{e}_{7}$ [1], where the multiplication rules between the basis are given as follows $[2,10]$ :

$$
\begin{gathered}
\mathrm{e}_{i} \mathrm{e}_{j}+\mathrm{e}_{j} \mathrm{e}_{i}=-2 \delta_{i j}, i, j=1, \ldots, 7, \\
\mathrm{e}_{0}^{2}=\mathrm{e}_{0}=1, \mathrm{e}_{i} \mathrm{e}_{0}=\mathrm{e}_{0} \mathrm{e}_{i}=\mathrm{e}_{i}, \mathrm{e}_{i}^{2}=-1, i=1, \ldots, 7 .
\end{gathered}
$$

For each $x \in O$, it can be written a $x=\sum_{i=0}^{7} x_{i} e_{i}\left(x_{i} \in \mathbb{R}\right)$ and its conjugate 


$$
\bar{x}=x_{0} e_{0}-\sum_{i=1}^{7} x_{i} e_{i}
$$

where $\quad \bar{e}_{0}=e_{0} \quad, \quad \bar{e}_{j}=-e_{j}(j=1, \ldots, 7) \quad . \quad$ Then $\overline{e_{i} e_{j}}=\overline{e_{j}} \overline{e_{i}}(\forall i, j=1, \ldots, 7)$ and $\overline{x y}=\bar{y} x(x, y \in O)$.

In order to illustrate the relationship between octonion multiplication, shown in Figure 1. Fano mnemonic where for simplicity we have used which consists of 7-points and 7-directed lines, then 7-points represent the standard basis for octonions.

Octonion multiplication are the nonassociative, noncommutative. But the subalgebra generated by any two elements is associative, namely, the octonions are alternative. So, for any $x, y, z \in O$, the associator $[\mathrm{x}, \mathrm{y}, \mathrm{z}]$ is defined by $[\mathrm{x}$, $\mathrm{y}, \mathrm{z}]=(\mathrm{xy}) \mathrm{z}-\mathrm{x}(\mathrm{yz})$.

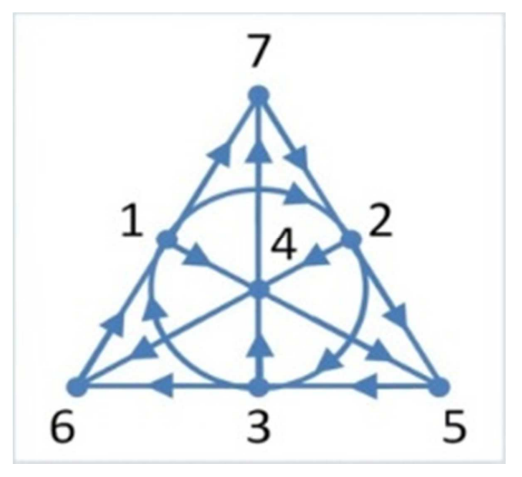

Figure 1. Fano Mnemonic.

Octonions obey some weakened associative laws, including the so-called Moufang identities, for any $x, y, z \in O$, it satisfies $[1,19]$.

$$
[x, y, z]=-[y, x, z]=[y, z, x],[x, x, y]=[x, \bar{x}, y]=0 .(x y x) z=x(y(x z)), z(x y x)=((z x) y) x, x(y z) x=(x y)(z x) .
$$

\section{Biregular Function}

The corresponding Dirac operator is defined as

$$
D=\sum_{i=0}^{7} e_{i} \partial_{x_{i}}
$$

More precisely,

$$
D f(x)=\sum_{i=0}^{7} \sum_{j=0}^{7} e_{i} e_{j} \partial_{x_{i}} f_{j}, f(x) D=\sum_{i=0}^{7} \sum_{j=0}^{7} e_{j} e_{i} \partial_{x_{i}} f_{j}
$$

for any $O$-valued function $f(x)=\sum_{j=0}^{7} e_{j} f_{j}(x)$ with $f_{j}(x)$ being real valued.

Definition 1: Let $\Omega \subset \mathbb{R}^{8}$ be a nonempty, open and connected set and $f \in C^{1}(\Omega, O)$. If for any $x \in \Omega$ we have

$$
D f(x)=0,(f(x) D=0) \text { in } \Omega,
$$

then the function is said to be left regular function (right regular function) in $\Omega$. In short, left regular function is also called regular function.

Definition 2: Suppose $\Omega=\Omega_{1} \times \Omega_{2}$ be a nonempty, open and connected set in $\mathbb{R}^{8} \times \mathbb{R}^{8}$ and $f \in C^{1}(\Omega, O)$. If for any $(x, y) \in \Omega$ we have

$$
\left\{\begin{array}{l}
D_{x} f(x, y)=0 \\
f(x, y) D_{y}=0
\end{array}\right.
$$

then the function $f$ is called as a biregular function in $\Omega$.

\section{Cauchy Integral Formula}

We introduce the Cauchy kernel, which satisfies the relation

$$
E_{1}(u-x)=\frac{1}{w_{8}} \frac{\overline{u-x}}{|u-x|^{8}}, E_{2}(v-y)=\frac{1}{w_{8}} \frac{\overline{v-y}}{|v-y|^{8}},
$$


Then

$$
\left\{\begin{array}{l}
D_{u} E_{1}(u-x)=E_{1}(u-x) D_{u}=0 \\
D_{v} E_{2}(v-y)=E_{2}(v-y) D_{v}=0
\end{array}\right.
$$

Where $w_{8}=\frac{\pi^{4}}{3}$ is the area of the unit sphere in $\mathbb{R}^{8}$.

For later use, we introduce volume element $d v_{y}=d y_{0} \wedge d y_{1} \wedge \ldots \wedge d y_{7}$ and surface measure

$$
\begin{gathered}
d \sigma_{y}=\sum_{i=0}^{7}(-1)^{i} e_{i} d \hat{y_{i}}=\sum_{i=0}^{7} e_{i} v_{i} d s_{y} \\
d \hat{y_{i}}=d y_{0} \wedge \ldots \wedge d y_{i-1} \wedge d y_{i+1} \ldots \wedge d y_{7}
\end{gathered}
$$

where

$$
d \hat{y_{i}}=d y_{0} \wedge \ldots \wedge d y_{i-1} \wedge d y_{i+1} \ldots \wedge d y_{7}
$$

stands the removal of $d y_{i}$ from $d v_{y}$ for $i=0,1, \ldots, 7$ and $v_{i}$ is $i$-th component of unit outward normal and $d s_{y}$ is the classical Lebesgue surface measure.

Theorem 1 (Stokes theorem 1)[12,17] Let $U \subset \mathbb{R}^{8}$ be open and let $S \subset U$ be an7-dimensional compact differentiable oriented manifold with boundary. Then for all $f, g \in C^{1}(U, O)$,

$$
\int_{S}\left\{(f D) g+f(D g)-\sum_{j=0}^{7}\left[\partial_{j} f, e_{j}, g\right]\right\} d v_{y}=\int_{\partial S} f\left(d \sigma_{y} g\right) .
$$

For convenience, we introduce the Stokes theorem2.

Theorem 2 (Stokes theorem 2) Let $U \subset \mathbb{R}^{8}$ be open and let $S \subset U$ be an 7-dimensional compact differentiable oriented manifold with boundary. Then for all $f, g \in C^{1}(U, O)$

$$
\int_{S}\left\{(f D) g+f(D g)+\sum_{j=0}^{7}\left[f, e_{j}, \partial_{j} g\right]\right\} d v_{y}=\int_{\partial S}\left(f d \sigma_{y}\right) g .
$$

Proof: Applying the divergence theorem, we know that

$$
\begin{aligned}
& \int_{\partial S}\left(f d \sigma_{y}\right) g=\int_{S} \sum_{j=0}^{7} \partial_{y_{j}}\left(f d \sigma_{y}\right) d y_{j} g+\left(f d \sigma_{y}\right) \sum_{j=0}^{7} \partial_{y_{j}} g d y_{j} \\
& =\int_{S}\left[\sum_{j=0}^{7} \partial_{y_{j}}\left(f \sum_{i=0}^{7}(-1)^{i} e_{i} d \hat{y_{i}}\right) d y_{j} g+\left(f \sum_{i=0}^{7}(-1)^{i} e_{i} d \hat{y_{i}}\right) \sum_{j=0}^{7} \partial_{y_{j}} g d y_{j}\right] \\
& =\int_{S}\left[\sum_{j=0}^{7} \partial_{y_{j}}\left(f \sum_{j=0}^{7}(-1)^{j} e_{j} d \hat{y_{j}}\right) d y_{j} g+\left(f \sum_{j=0}^{7}(-1)^{j} e_{j} d \hat{y_{j}}\right) \sum_{j=0}^{7} \partial_{y_{j}} g d y_{j}\right] \\
& =\int_{S}\left[(f D) g+\sum_{j=0}^{7}\left(f e_{j}\right) \partial_{y_{j}} g\right] d v_{y} \\
& =\int_{S}\left\{(f D) g+f(D g)+\sum_{j=0}^{7}\left[f, e_{j}, \partial_{y_{j}} g\right]\right\} d v_{y} .
\end{aligned}
$$

Lemma $1[16]$ For any $x, u \in O, x \neq u$, 


$$
\sum_{j=0}^{7}\left[\partial_{u_{j}} E_{1}(u-x), e_{j}, f(x, u)\right]=0, x \neq u
$$

Proof: Since

$$
E_{1}(u-x)=\frac{1}{w_{8}} \frac{\overline{u-x}}{|u-x|^{8}}
$$

and

$$
\partial_{u_{j}} \frac{\overline{u-x}}{|u-x|^{8}}=\frac{\overline{e_{j}}}{|u-x|^{8}}-8\left(u_{j}-x_{j}\right) \frac{\overline{u-x}}{|u-x|^{10}},
$$

we get

$$
\begin{aligned}
& \sum_{j=0}^{7}\left[\partial_{u_{j}} \frac{\overline{u-x}}{|u-x|^{8}}, e_{j}, f(x, u)\right] \\
= & \sum_{j=0}^{7}\left[\frac{\overline{e_{j}}}{|u-x|^{8}}-8\left(u_{j}-x_{j}\right) \frac{\overline{u-x}}{|u-x|^{10}}, e_{j}, f(x, u)\right] \\
= & \sum_{j=0}^{7}\left[\frac{\overline{e_{j}}}{|u-x|^{8}}, e_{j}, f(x, u)\right]-\sum_{j=0}^{7}\left[8\left(u_{j}-x_{j}\right) \frac{\overline{u-x}}{|u-x|^{10}}, e_{j}, f(x, u)\right] \\
= & \sum_{j=0}^{7} \frac{1}{|u-x|^{8}}\left[\overline{e_{j}}, e_{j}, f(x, u)\right]-\sum_{j=0}^{7} \frac{8}{|u-x|^{10}}[\overline{u-x}, u-x, f(x, u)] \\
= & 0,
\end{aligned}
$$

by the weak form of associativity (3) in the last step.

Theorem 3 (Cauchy integral formula) Suppose $\Omega=\Omega_{1} \times \Omega_{2} \subset \mathbb{R}^{8} \times \mathbb{R}^{8}$ is nonempty, connected and open set. $\partial \Omega_{1}, \partial \Omega_{2}$ are differentiable, oriented and compact Lyapunov surface, if $f$ is biregular function in $\Omega$, then

$$
\int_{\partial \Omega_{1} \times \partial \Omega_{2}}\left\{\left[E_{1}(u-x)\left(d \sigma_{u} f(u, v)\right)\right] d \sigma_{v}\right\} E_{2}(v-y)=\left\{\begin{array}{l}
f(x, y),(x, y) \in \Omega \\
0,(x, y) \notin \bar{\Omega}
\end{array}\right.
$$

Proof: When $(x, y) \notin \bar{\Omega}$,

$$
\begin{aligned}
& \int_{\partial \Omega_{1} \times \partial \Omega_{2}}\left\{\left[E_{1}(u-x)\left(d \sigma_{u} f(u, v)\right)\right] d \sigma_{v}\right\} E_{2}(v-y) \\
& =\int_{\partial \Omega_{2}}\left\{\left[\int_{\partial \Omega_{1}} E_{1}(u-x)\left(d \sigma_{u} f(u, v)\right)\right] d \sigma_{v}\right\} E_{2}(v-y) \\
& =\int_{\partial \Omega_{2}}\left\{\int_{\Omega_{1}}\left[\left(E_{1}(u-x) D_{u}\right) f(u, v)+E_{1}(u-x)\left(D_{u} f(u, v)\right)-\sum_{j=0}^{7}\left[\partial u_{j} E_{1}(u-x), e_{j}, f(x, u)\right] d v_{u} d \sigma_{v}\right\} E_{2}(v-y)\right. \\
& =0 .
\end{aligned}
$$

The first term of the above proof is due to the nature of kernel function $E_{1}(u-x) D_{u}=0$. Since $f$ is a biregular function $D_{u} f(u, v)=0$, then the second term vanishes, and $\left[\partial u_{j} E_{1}(u-x), e_{j}, f(x, u)\right]=0$ by Lemma 1 in the last term.

When $\quad(x, y) \in \Omega$, we construct a 7-dimensional hyspersphere $\quad B_{1}(x, \delta)=\left\{u \mid \sum_{i=0}^{7}\left(u_{i}-x_{i}\right)^{2} \leq \delta^{2}\right\} \subset \Omega_{1}$, 
$B_{2}(y, \delta)=\left\{v \mid \sum_{i=0}^{7}\left(v_{i}-y_{i}\right)^{2} \leq \delta^{2}\right\} \subset \Omega_{2}$, then

$$
\begin{aligned}
& \int_{\partial \Omega_{1} \times \partial \Omega_{2}}\left\{\left[E_{1}(u-x)\left(d \sigma_{u} f(u, v)\right)\right] d \sigma_{v}\right\} E_{2}(v-y) \\
& =\int_{\partial B_{1}(x, \delta) \times \partial B_{2}(y, \delta)}\left\{\left[E_{1}(u-x)\left(d \sigma_{u} f(u, v)\right)\right] d \sigma_{v}\right\} E_{2}(v-y) \\
& =\theta(\delta) .
\end{aligned}
$$

In the following proof, we will get $\lim _{\delta \rightarrow 0} \theta(\delta)=f(x, y)$.

$$
\begin{aligned}
\theta(\delta)= & \int_{\partial B_{2}(y, \delta)}\left\{\left[\int_{\partial B_{1}(x, \delta)} E_{1}(u-x)\left(d \sigma_{u} f(u, v)\right)\right] d \sigma_{v}\right\} E_{2}(v-y) \text {. Using the Stokes theorem 1, we thus obtain } \\
& \left.\frac{1}{w_{8} w_{8}} \frac{1}{\delta^{16}} \int_{\partial B_{2}(y, \delta)}\left\{\int_{B_{1}(x, \delta)}\left[\overline{(u-x} D_{u}\right) f(u, v)+\overline{u-x}\left(D_{u} f(u, v)\right)-\sum_{j=0}^{7}\left[\partial u_{j} \overline{u-x}, e_{j}, f(u, v)\right]\right] d v_{u} d \sigma_{v}\right\} \overline{v-y} \\
= & \left.\frac{1}{w_{8} w_{8}} \frac{1}{\delta^{16}} \int_{\partial B_{2}(y, \delta)}\left\{\int_{B_{1}(x, \delta)}\left[\overline{(u-x} D_{u}\right) f(u, v)+\overline{u-x}\left(D_{u} f(u, v)\right)-\sum_{j=0}^{7}\left[\overline{e_{j}}, e_{j}, f(u, v)\right]\right] d v_{u} d \sigma_{v}\right\} \overline{v-y} .
\end{aligned}
$$

The first term above $\overline{u-x} D_{u}=8$. The other two terms vanishes, according to definition 2 and the weak form of associativity (3), we get

$$
\frac{1}{w_{8} w_{8}} \frac{8}{\delta^{16}} \int_{B_{1}(x, \delta)}\left[\int_{\partial B_{2}(y, \delta)}\left(f(u, v) d \sigma_{v}\right) \overline{v-y}\right] d v_{u} .
$$

By Stokes theorem 2 in equation (21), we thus obtain

$$
\begin{aligned}
& \frac{1}{w_{8} w_{8}} \frac{8}{\delta^{16}} \int_{B_{1}(x, \delta)}\left\{\int_{B_{2}(y, \delta)}\left[\left(f(u, v) D_{v}\right) \overline{v-y}+f(u, v)\left(D_{v} \overline{v-y}\right)+\sum_{j=0}^{7}\left[f(u, v), e_{j}, \partial v_{j} \overline{v-y}\right] d v_{v}\right\} d v_{u}\right. \\
& =\frac{1}{w_{8} w_{8}} \frac{8}{\delta^{16}} \int_{B_{1}(x, \delta)}\left\{\int_{B_{2}(y, \delta)}\left[\left(f(u, v) D_{v}\right) \overline{v-y}+f(u, v)\left(D_{v} \overline{v-y}\right)+\sum_{j=0}^{7}\left[f(u, v), e_{j}, e_{j}\right]\right] d v_{v}\right\} d v_{u} \\
& =\frac{1}{w_{8} w_{8}} \frac{64}{\delta^{16}} \int_{B_{1}(x, \delta)}\left[\int_{B_{2}(y, \delta)} f(u, v) d v_{v}\right] d v_{u} .
\end{aligned}
$$

The proof is similar to (20).

Therefore, for sufficiently small $\delta$, we have $f(u, v)=f(u, y)+\theta(\delta), f(u, y)=f(x, y)+\theta(\delta)$, then

$$
\begin{aligned}
& \lim _{\delta \rightarrow 0} \theta(\delta)=\frac{1}{w_{8} w_{8}} \frac{64}{\delta^{8}} \int_{B_{1}(x, \delta)}\left[\int_{B_{2}(y, \delta)}(f(u, y)+\theta(\delta)) d v_{v}\right] d v_{u} \\
& =\frac{8}{\delta^{8}} \int_{B_{1}(x, \delta)} f(u, y) d v_{u} \\
& =\frac{8}{\delta^{8}} \int_{B_{1}(x, \delta)}(f(x, y)+\theta(\delta)) d v_{u} \\
& =f(x, y) .
\end{aligned}
$$

Then the proof of the result is completed.

Corollary: Let $\Omega=\Omega_{1} \times \Omega_{2}$ be as stated above, $\partial \Omega_{1}, \partial \Omega_{2}$ are differentiable, oriented and compact Lyapunov surface, if $f$ is biregular function in $\Omega$, then we have 


$$
\int_{\partial \Omega_{1} \times \partial \Omega_{2}}\left\{\left[E_{1}(u-x)\left(d \sigma_{u}\right)\right] d \sigma_{v}\right\} E_{2}(v-y)=\left\{\begin{array}{l}
1, f o r(x, y) \in \Omega, \\
0, \text { for }(x, y) \notin \bar{\Omega} .
\end{array}\right.
$$

Theorem 4 (Mean value theorem) Let $B(x, \delta) \subset \mathbb{R}^{8}, B(y, \delta) \subset \mathbb{R}^{8}$ and $f$ is a biregular function in $B\left(x, \delta_{1}\right) \times B\left(y, \delta_{2}\right)$, we have

$$
f(x, y)=\frac{1}{\delta_{1}^{8} v_{8} \delta_{2}^{8} v_{8}} \int_{B\left(x, \delta_{1}\right) \times B\left(y, \delta_{2}\right)} f(u, y) d v_{u} d v_{v} .
$$

Proof: Applying Cauchy integral formula, we know

$$
\begin{aligned}
f(x, y) & =\frac{1}{\delta_{1}^{8} w_{8} \delta_{2}^{8} w_{8}} \int_{\partial B\left(x, \delta_{1}\right) \times \partial B\left(y, \delta_{2}\right)}\left\{\left[\overline{u-x}\left(d \sigma_{u} f(u, v)\right)\right] d \sigma_{v}\right\} \overline{v-y} \text {. Let's use Stokes theorem 1, we have } \\
& \frac{1}{\delta_{1}^{8} w_{8} \delta_{2}^{8} w_{8}} \int_{\partial B\left(y, \delta_{2}\right)}\left\{\int_{B\left(x, \delta_{1}\right)}\left[\overline{(u-x} D_{u}\right) f(u, v)+\overline{u-x}\left(D_{u} f(u, v)\right)-\sum_{j=0}^{7}\left[\partial u_{j} \overline{u-x}, e_{j}, f(u, v)\right] d v_{u} d \sigma_{v} \overline{v-y}\right. \\
& \left.=\frac{1}{\delta_{1}^{8} w_{8} \delta_{2}^{8} w_{8}} \int_{\partial B\left(y, \delta_{2}\right)}\left\{\int_{B\left(x, \delta_{1}\right)}\left[\overline{(u-x} D_{u}\right) f(u, v)+\overline{u-x}\left(D_{u} f(u, v)\right)-\sum_{j=0}^{7}\left[\overline{e_{j}}, e_{j}, f(u, v)\right]\right] d v_{u} d \sigma_{v}\right\} \overline{v-y},
\end{aligned}
$$

By the weak form of associativity (3) in the last term, and the other two terms are due to

$D_{u} f(u, v)=0, f(u, v) D_{v}=0 ; \overline{u-x} D_{u}=8, D_{v} \overline{v-y}=8$, we get

$$
\frac{1}{\delta_{1}^{8} w_{8} \delta_{2}^{8} w_{8}} \int_{\partial B\left(y, \delta_{2}\right)}\left\{\int_{B\left(x, \delta_{1}\right)}[8 f(u, v)] d v_{u} d \sigma_{v}\right\} \overline{v-y}=\frac{8}{\delta_{1}^{8} w_{8} \delta_{2}^{8} w_{8}} \int_{B\left(x, \delta_{1}\right)}\left\{\int_{\partial B\left(y, \delta_{2}\right)}\left(f(u, v) d \sigma_{v}\right) \overline{v-y}\right\} d v_{u} .
$$

By Stokes theorem 2, we have

$$
\begin{aligned}
& \frac{8}{\delta_{1}^{8} w_{8} \delta_{2}^{8} w_{8}} \int_{B\left(x, \delta_{1}\right) \times B\left(y, \delta_{2}\right)}\left\{\left(f(u, v) D_{v}\right) \overline{v-y}+f(u, v)\left(D_{v} \overline{v-y}\right)+\sum_{j=0}^{7}\left[f(u, v), e_{j}, \partial v_{j} \overline{v-y}\right]\right\} d v_{u} d v_{v} \\
& =\frac{8}{\delta_{1}^{8} w_{8} \delta_{2}^{8} w_{8}} \int_{B\left(x, \delta_{1}\right) \times B\left(y, \delta_{2}\right)}\left\{\left(f(u, v) D_{v}\right) \overline{v-y}+f(u, v)\left(D_{v} \overline{v-y}\right)+\sum_{j=0}^{7}\left[f(u, v), e_{j}, e_{j}\right]\right\} d v_{u} d v_{v} \\
& =\frac{64}{\delta_{1}^{8} w_{8} \delta_{2}^{8} w_{8}} \int_{B\left(x, \delta_{1}\right) \times B\left(y, \delta_{2}\right)} f(u, v) d v_{u} d v_{v} \\
& =\frac{1}{\delta_{1}^{8} w_{8} \delta_{2}^{8} w_{8}} \int_{B\left(x, \delta_{1}\right) \times B\left(y, \delta_{2}\right)} f(u, v) d v_{u} d v_{v} .
\end{aligned}
$$

The proof is similar to the above manner. Hence $v_{8}=\frac{w_{8}}{8}$ is volume of the 7-dimensional unit ball.

TUTE (KYQD14041, KJ15-18).

\section{Conclusion}

In this paper, using the methods in dealing with the Cauchy integral formula of biregular function in Clifford analysis and regular function in octonionic analysis, we obtain the Cauchy integral formula and mean value theorem for biregular function in octonionic analysis.

\section{Funding}

The work was supported by the National Natural Science Foundation of China (No. 11601390) and the foundation of

\section{Acknowledgements}

The authors would like to thank the referee for his valuable suggestions, which will help me to improve the presentation of the paper.

\section{References}

[1] Baez, J. C. (2002) The octonions [J]. Bull Amer Math Soc, 39, 145-205.

[2] Baez, J. C. (2005) On quaternions and octonions: Their geometry, arithmetic and symmetry [J]. Bull Amer Math Soc, 42, 229-243. 
[3] Mironov, V. L. and Mironov, S. V. (2009) Octonic representation of electromagnetic field equation [J]. J Math Physics, 50, 1-10.

[4] Okubo, S. (1995) Introduction to Octonion and Other Non-Associative Algebras in Physics [M]. Cam-bridge University Press, New York.

[5] Bossard, G. (2012) Octonionic black holes [J]. Journal of High Energy Physics, 5, 1-78.

[6] Cherkis, S. A. (2015) Octonions, monopoles and knots [J]. Lett Math Phys, 105 (5), 641-659.

[7] Borstena, L. and Dahanayakea, D. and Duffa, M. J. and Ebrahima, H. and Rubensa W. (2009) Black holes, qubits and octonions [J]. Physics Reports, 471 (3-4), 113-219.

[8] Wang, H. M. and Wang, W. (2014) On octonionic regular functions and szego projection on the octonionic Heisenberg group [J]. Complex Anal Oper Theory, 8, 1285-1324.

[9] Calin, O. and Chang, D. C. and Markina, I. (2009) Geometric analysis on $\mathrm{H}$-type groups related to division algebras [J]. Math Nachr, 282 (1), 44-68.

[10] Li, X. M. (1998) Octonion analysis, PhD thesis, Peking University, Beijing.

[11] Baez, J. C. and Huerta, J. (2011) The strangest numbers in string theory $[\mathrm{J}]$. Sci Am, 304 (5), 60-65.
[12] Li, X. M. and Zhao, K. and Peng, L. Z. (2002) The Cauchy integral formulas on the octonions [J]. Bull Belg Math Soc Simon Stevin, 9 (1), 47-64.

[13] Peng, L. Z. and Zhao, J. M. (2000) Hardy space and Bergman space on the Octonions [J]. Approximation Theory and Its Applications, 16 (3), 72-84.

[14] Ludkovsky, S. V. (2010) Feynman integration over octonions with application to quantum mechanics [J]. Math Meth Appl Sci, 33 (9), 1148-1173.

[15] Hahn, S. L. and Snopek, K. M. (2011) The unified theory of n-dimensional complex and hypercomplex analytic signals [J]. Bull Pol Ac Tech, 59 (2), 167-181.

[16] Wang, H. Y. and Bian, X. L. (2017) The right inverse of Dirac operator in octonionic space [J]. Journal of Geometry and Physics, 119, 139-145.

[17] Wang, H. Y. and Ren, G. B. (2014) Octonion analysis of several variables [J]. Communications in Mathematics and Statistics, 2, 163-185.

[18] Wang, J. X. and Li, X. M. (2018) The octonionic Bergman kernel for the unit ball [J]. Adv Appl Clifford Algebras, 28, 60.

[19] Jacobson, N. (1985) Basic Algebra I, W H Freeman and Company, New York. 